\title{
On global inversion of homogeneous maps
}

\author{
Michael Ruzhansky • Mitsuru Sugimoto
}

Received: 27 September 2014 / Accepted: 3 October 2014 / Published online: 29 October 2014 (C) The Author(s) 2014. This article is published with open access at SpringerLink.com

\begin{abstract}
In this note we prove a global inverse function theorem for homogeneous mappings on $\mathbb{R}^{n}$. The proof is based on an adaptation of the Hadamard's global inverse theorem which provides conditions for a function to be globally invertible on $\mathbb{R}^{n}$. For the latter adaptation, we give a short elementary proof assuming a topological result.
\end{abstract}

Keywords Inverse function theorem - Homogeneous mappings · Global inverse

Mathematics Subject Classification Primary 26B10; Secondary 26B05 $\cdot$ 26-01

\section{Introduction}

The purpose of this note is to prove a global inverse function for homogeneous mappings on $\mathbb{R}^{n}$. The main difficulty is in the fact that such mappings are not smooth at the origin and thus, the known global inverse function theorems on $\mathbb{R}^{n}$ are not readily applicable. The main motivation for studying such inverses are applications to the global invertibility of Hamiltonian flows, and further applications to construction of

Communicated by Ari Laptev.

M. Ruzhansky was supported by the EPSRC Leadership Fellowship EP/G007233/1 and by EPSRC Grant EP/K039407/1.

M. Ruzhansky ( $\square)$

Department of Mathematics, Imperial College London, 180 Queen's Gate, London SW7 2AZ, UK e-mail: m.ruzhansky@imperial.ac.uk

\section{Sugimoto}

Graduate School of Mathematics, Nagoya University, Furocho Chikusa-ku, Nagoya 464-8602, Japan e-mail: sugimoto@math.nagoya-u.ac.jp 
suitable phase functions of Fourier integral operators, a topic that will be addressed elsewhere.

Thus, our aim here is to establish the existence and the following properties for the global inverses of homogeneous mappings.

Theorem 1.1 Let $n \geq 3$ and $1 \leq k \leq \infty$. Let $f: \mathbb{R}^{n} \backslash 0 \rightarrow \mathbb{R}^{n}$ be a positively homogeneous mapping of order $x>0$, i.e.

$$
f(\tau \xi)=\tau^{\varkappa} f(\xi) \text { for all } \tau>0, \xi \neq 0 .
$$

Assume that $f \in C^{k}\left(\mathbb{R}^{n} \backslash 0\right)$ and that its Jacobian never vanishes on $\mathbb{R}^{n} \backslash 0$. Then $f$ is bijective from $\mathbb{R}^{n} \backslash 0$ to $\mathbb{R}^{n} \backslash 0$, its global inverse satisfies $f^{-1} \in C^{k}\left(\mathbb{R}^{n} \backslash 0\right)$ and is positively homogeneous of order $1 / \varkappa$. Moreover, if we extend $f$ to $\mathbb{R}^{n}$ by setting $f(0)=0$, the extension is a global homeomorphism on $\mathbb{R}^{n}$.

We remark that for $n=2$ the conclusion is not always true because, for example, the Jacobian of $f(x, y)=\left(x^{2}-y^{2}, 2 x y\right)$ never vanishes on $\mathbb{R}^{2} \backslash 0$ but $f$ is not globally invertible since $f(x, y)=f(-x,-y)$.

Our proof is based on the application of an adaptation of the Hadamard global inverse theorem. Thus, the second aim of this note is to give a short elementary proof for it assuming a well-known topological result (very likely also known to Hadamard).

This kind of global inverse function theorem is a classical subject and of independent interest, but sometimes it also plays an important role when we discuss the global $L^{2}$ boundedness of oscillatory integrals. For example, Asada-Fujiwara [1] established this boundedness based on the global invertibility of maps defined by phase functions which are smooth everywhere. Generalising such results to the case of homogeneous phase as in Theorem 1.1 is also important but it is not straightforward because of the singularity at the origin. The global $L^{2}$ boundedness of oscillatory integrals with phases as in Theorem 1.1 has been analysed by the authors in [7] and applied to questions of global smoothing for partial differential equations in [8]. The application of Theorem 1.1 to the global analysis of hyperbolic partial differential equations and the corresponding Hamiltonian flows will appear elsewhere.

\section{Global inverses}

Let us start with a topological result we assume. A differentiable map between manifolds is called a $C^{1}$-diffeomorphism if it is one-to-one and its inverse is also differentiable. A mapping $f$ is called proper if $f^{-1}(K)$ is compact whenever $K$ is compact.

Theorem 2.1 Let $M$ and $N$ be connected, oriented, d-dimensional $C^{1}$-manifolds, without boundary. Let $f: M \rightarrow N$ be a proper $C^{1}$-map such that the Jacobian $J(f)$ never vanishes. Then $f$ is surjective. If $N$ is simply connected in addition, then $f$ is also injective.

This fact was known to Hadamard, but a rigorous proof for surjectivity can be found in [6]. As for the injectivity, a precise proof can be found in [2, Sect. 3]. We remark that it follows in principle from the general fact that a proper submersion between 
smooth manifolds without boundary is a fibre bundle, meaning in our setting that it is a covering map. Since a simply connected manifold is its own universal covering space, it implies that $f$ is a diffeomorphism.

We will mostly discuss the mappings $f: \mathbb{R}^{n} \rightarrow \mathbb{R}^{n}$, in which case we denote its Jacobian by $J(f):=\operatorname{det} D f:=\operatorname{det}\left(\partial f_{i} / \partial x_{j}\right)$. The Hadamard global inverse function theorem states:

Theorem 2.2 A $C^{1}$-map $f: \mathbb{R}^{n} \rightarrow \mathbb{R}^{n}$ is a $C^{1}$-diffeomorphism if and only if the Jacobian det $D f(w)$ never vanishes and $|f(y)| \rightarrow \infty$ whenever $|y| \rightarrow \infty$.

This theorem goes back to Hadamard [3-5]. In fact, in 1972 W. B. Gordon wrote "This theorem goes back at least to Hadamard, but it does not appear to be 'wellknown'. Indeed, I have found that most people do not believe it when they see it and that the skepticism of some persists until they see two proofs." The reason behind this is that while we know that the function is locally a $C^{1}$-diffeomorphism by the usual local inverse function theorem, the condition that $|f(y)| \rightarrow \infty$ as $|y| \rightarrow \infty$, guarantees that the function is both injective and, more importantly, surjective on the whole of $\mathbb{R}^{n}$. And indeed, W. B. Gordon proceeds in [2] by giving two different proofs for it, for $C^{2}$ and for $C^{1}$ mappings.

It turns out that for dimensions $n \geq 3$ the conclusion remains still valid even if we relax the assumption, in some sense rather substantially, almost removing it at a finite number of points. While often this is not the case (the famous one being the 'hairy ball theorem', which fails completely if we assume that the vector field may be not differentiable at one point), the theory of covering spaces assures that it is the case here. In fact, here we have the following:

Theorem 2.3 Let $n \geq 3$. Let $a \in \mathbb{R}^{n}$. Let $f: \mathbb{R}^{n} \rightarrow \mathbb{R}^{n}$ be such that $f$ is $C^{1}$ on $\mathbb{R}^{n} \backslash\{a\}$, with det $D f \neq 0$ on $\mathbb{R}^{n} \backslash\{a\}$, and that $f$ is continuous at $a$. Let $b:=f(a)$, and assume that $f\left(\mathbb{R}^{n} \backslash\{a\}\right) \subset \mathbb{R}^{n} \backslash\{b\}$ and that $|y| \rightarrow \infty$ implies $|f(y)| \rightarrow \infty$. Then the mapping $f: \mathbb{R}^{n} \rightarrow \mathbb{R}^{n}$ is a global homeomorphism and its restriction $f: \mathbb{R}^{n} \backslash\{a\} \rightarrow \mathbb{R}^{n} \backslash\{b\}$ is a global $C^{1}$-diffeomorphism.

While general topological considerations as outlined above are possible here, we prefer to also give an elementary proof in Sect. 3 of the fact that Theorem 2.1 implies Theorem 2.3, also noting that exactly the same proof yields the following further version:

Theorem 2.4 Let $n \geq 3$. Let $A \subset \mathbb{R}^{n}$ be a closed set. Let $f: \mathbb{R}^{n} \rightarrow \mathbb{R}^{n}$ be such that $f$ is $C^{1}$ on $\mathbb{R}^{n} \backslash A$, with $\operatorname{det} D f \neq 0$ on $\mathbb{R}^{n} \backslash A$, that $f$ is continuous and injective on $A$, and that $\mathbb{R}^{n} \backslash f(A)$ is simply connected. Assume that $f\left(\mathbb{R}^{n} \backslash A\right) \subset \mathbb{R}^{n} \backslash f(A)$ and that $|y| \rightarrow \infty$ implies $|f(y)| \rightarrow \infty$. Then the mapping $f: \mathbb{R}^{n} \rightarrow \mathbb{R}^{n}$ is a global homeomorphism and its restriction $f: \mathbb{R}^{n} \backslash A \rightarrow \mathbb{R}^{n} \backslash f(A)$ is a global $C^{1}$ diffeomorphism.

Given Theorem 2.3, we can apply it to derive Theorem 1.1:

Proof of Theorem 1.1 First, let us extend $f$ to $\mathbb{R}^{n}$ by setting $f(0)=0$ and show that $f$ is continuous at 0 . We observe that $f\left(\mathbb{S}^{n-1}\right)$ has a finite maximum. Let $\xi_{j} \rightarrow 0$, and $\xi_{j} \neq 0$ for all $j$. Then 


$$
\left|f\left(\xi_{j}\right)\right|=\left|\xi_{j}\right|^{\varkappa}\left|f\left(\frac{\xi_{j}}{\left|\xi_{j}\right|}\right)\right| \leq C\left|\xi_{j}\right|^{\varkappa} \rightarrow 0
$$

so that $f$ is continuous at 0 .

Let us now check that other conditions of Theorem 2.3 are satisfied. We observe that $f\left(\mathbb{S}^{n-1}\right)$ has a positive minimum $\min _{|\xi|=1}|f(\xi)|=c_{0}>0$. Indeed, if $f(\omega)=0$ for some $\omega \in \mathbb{S}^{n-1}$, then $f(t \omega)\left(=t^{\kappa} f(\omega)\right)=0$ for any $t>0$. Differentiating it in $t$, we have $\omega=0$ since the Jacobian of $f$ never vanishes on $\mathbb{R}^{n} \backslash 0$, which is a contradiction. Then we have

$$
|f(\xi)|=|\xi|^{\varkappa}\left|f\left(\frac{\xi}{|\xi|}\right)\right| \geq c_{0}|\xi|^{\varkappa}, \quad \xi \neq 0,
$$

which induces that $f\left(\mathbb{R}^{n} \backslash 0\right) \subset \mathbb{R}^{n} \backslash 0$ and that $|y| \rightarrow \infty$ implies $|f(y)| \rightarrow \infty$. Therefore, by Theorem 2.3, $f: \mathbb{R}^{n} \rightarrow \mathbb{R}^{n}$ is a homeomorphism, and $f^{-1}$ is $C^{k}$ on $\mathbb{R}^{n} \backslash 0$ by the usual local inverse function theorem. Let us finally show that $f^{-1}$ is positively homogeneous of order $1 / \varkappa$. Indeed, for every $\tau>0$ and $\xi \neq 0$ we have $f^{-1}\left(\tau^{\varkappa} f(\xi)\right)=f^{-1}(f(\tau \xi))=\tau \xi$. Since $f$ is invertible, $\eta=f(\xi) \neq 0$, and we have $f^{-1}\left(\tau^{\varkappa} \eta\right)=\tau f^{-1}(\eta)$, or $f^{-1}(\tau \eta)=\tau^{1 / \varkappa} f^{-1}(\eta)$.

\section{Proofs}

First we observe that in the setting of Theorem 2.3, by translation (by $a$ ) in $x$ and by subtracting $b$ from $f$, we may assume without loss of generality that $a=b=0$. To prove Theorem 2.3, we start with preliminary statements.

Lemma 3.1 Let $F \subset \mathbb{R}^{n} \backslash 0$. Then $F$ is compact in $\mathbb{R}^{n} \backslash 0$ if and only if it is compact in $\mathbb{R}^{n}$.

Proof Assume that $F \subset \mathbb{R}^{n} \backslash 0$ is compact in $\mathbb{R}^{n} \backslash 0$. Let $F \subset \bigcup_{\alpha} V_{\alpha}$ for a family of sets $V_{\alpha}$ which are open in $\mathbb{R}^{n}$. Then

$$
F \subset\left(\bigcup_{\alpha} V_{\alpha}\right) \cap\left(\mathbb{R}^{n} \backslash 0\right)=\bigcup_{\alpha}\left(V_{\alpha} \cap\left(\mathbb{R}^{n} \backslash 0\right)\right),
$$

so that $F$ is covered by a family of sets $V_{\alpha} \cap\left(\mathbb{R}^{n} \backslash 0\right)$ which are open in $\mathbb{R}^{n} \backslash 0$. Since $F$ is compact in $\mathbb{R}^{n} \backslash 0$, there is a finite subfamily $V_{j}, j=1, \ldots, m$, such that

$$
F \subset \bigcup_{j=1}^{m}\left(V_{j} \cap\left(\mathbb{R}^{n} \backslash 0\right)\right)=\left(\bigcup_{j=1}^{m} V_{j}\right) \cap\left(\mathbb{R}^{n} \backslash 0\right) .
$$

Hence $F \subset \bigcup_{j=1}^{m} V_{j}$, so that $F$ is compact in $\mathbb{R}^{n}$.

Conversely, assume that $F \subset \mathbb{R}^{n} \backslash 0$ is compact in $\mathbb{R}^{n}$, and let $F \subset \bigcup_{\alpha} U_{\alpha}$, for a family of sets $U_{\alpha} \subset \mathbb{R}^{n} \backslash 0$ which are open in $\mathbb{R}^{n} \backslash 0$. Then $U_{\alpha}=V_{\alpha} \cap\left(\mathbb{R}^{n} \backslash 0\right)$, for 
some $V_{\alpha}$ open in $\mathbb{R}^{n}$. Hence $F \subset \bigcup_{\alpha} V_{\alpha}$, and by compactness of $F$ in $\mathbb{R}^{n}$, there is a finite subcovering $F \subset \bigcup_{j=1}^{m} V_{j}$. Since $F \subset \mathbb{R}^{n} \backslash 0$, we have

$$
F \subset\left(\bigcup_{j=1}^{m} V_{j}\right) \cap\left(\mathbb{R}^{n} \backslash 0\right)=\bigcup_{j=1}^{m}\left(V_{j} \cap\left(\mathbb{R}^{n} \backslash 0\right)\right)=\bigcup_{j=1}^{m} U_{j},
$$

which proves that $F$ is compact in $\mathbb{R}^{n} \backslash 0$.

We recall that a mapping $f$ is called proper if $f^{-1}(K)$ is compact whenever $K$ is compact.

Lemma 3.2 Let $f: \mathbb{R}^{n} \rightarrow \mathbb{R}^{n}$ be proper and such that $f(0)=0$ and $f\left(\mathbb{R}^{n} \backslash 0\right) \subset$ $\mathbb{R}^{n} \backslash 0$. Then the restriction $f: \mathbb{R}^{n} \backslash 0 \rightarrow \mathbb{R}^{n} \backslash 0$ is proper.

Proof Let $K \subset \mathbb{R}^{n} \backslash 0$ be compact in $\mathbb{R}^{n} \backslash 0$. By Lemma 3.1 it is compact in $\mathbb{R}^{n}$, and, since $f$ is proper, the set $f^{-1}(K)$ is compact in $\mathbb{R}^{n}$. We notice that if $0 \in f^{-1}(K)$ then we would have $0=f(0) \in K$, which is impossible since $K \subset \mathbb{R}^{n} \backslash 0$. Hence $f^{-1}(K) \subset \mathbb{R}^{n} \backslash 0$, and by Lemma 3.1 again, the set $f^{-1}(K)$ is compact in $\mathbb{R}^{n} \backslash 0$. Hence the restriction $f: \mathbb{R}^{n} \backslash 0 \rightarrow \mathbb{R}^{n} \backslash 0$ is proper.

Lemma 3.3 Let $f: \mathbb{R}^{n} \rightarrow \mathbb{R}^{n}$ be continuous everywhere. Then $f$ is proper if and only if $|y| \rightarrow \infty$ implies $|f(y)| \rightarrow \infty$.

Proof We show the if part. Let $K \subset \mathbb{R}^{n}$ be compact. Then it is closed and hence $f^{-1}(K) \subset \mathbb{R}^{n}$ is closed. Suppose $f^{-1}(K)$ is not bounded. Then there is a sequence $y_{j} \in f^{-1}(K)$ such that $\left|y_{j}\right| \rightarrow \infty$. Hence $f\left(y_{j}\right) \in K$ and also $\left|f\left(y_{j}\right)\right| \rightarrow \infty$ by the assumption on $f$, which yields a contradiction with the boundedness of $K$. The converse implication is clearly also true.

Lemma 3.4 Let $f: \mathbb{R}^{n} \rightarrow \mathbb{R}^{n}$ be proper, injective, and continuous. Then $f$ is an open map.

Proof Let us assume that $f(U)$ is not open for an open subset $U \subset \mathbb{R}^{n}$. Then there is a point $a \in U$ such that $f(a)$ is on the boundary of $f(U)$, and we can construct a sequence $y_{j} \in \mathbb{R}^{n} \backslash U$ such that $f\left(y_{j}\right) \rightarrow f(a)$. Since $f$ is proper, there exists a subsequence $y_{j}^{\prime}$ which converges to some point $b \in \mathbb{R}^{n} \backslash U$. Note that $b \neq a$. Since $f$ is continuous, $f\left(y_{j}^{\prime}\right) \rightarrow f(b)$, but we also have $f\left(y_{j}^{\prime}\right) \rightarrow f(a)$ which contradicts to the fact that $f$ is injective.

The following result is a straight forward consequence of Theorem 2.1.

Corollary 3.5 Let $n \geq 2$. Let $f: \mathbb{R}^{n} \rightarrow \mathbb{R}^{n}$ be proper and such that and $f(0)=0$ and $f\left(\mathbb{R}^{n} \backslash 0\right) \subset \mathbb{R}^{n} \backslash 0$. Moreover, assume that $f$ is $C^{1}$ on $\mathbb{R}^{n} \backslash 0$, with $\operatorname{det} D f \neq 0$ on $\mathbb{R}^{n} \backslash 0$. Then the restriction $f: \mathbb{R}^{n} \backslash 0 \rightarrow \mathbb{R}^{n} \backslash 0$ is surjective. If $n \geq 3$ in addition, $f: \mathbb{R}^{n} \backslash 0 \rightarrow \mathbb{R}^{n} \backslash 0$ is also injective.

Proof By Lemma 3.2, the restriction of $f$ is a proper map from $M=\mathbb{R}^{n} \backslash 0$ to $N$ $=\mathbb{R}^{n} \backslash 0$. Note that $N$ is simply connected if $n \geq 3$. Then Theorem 2.1 implies the statement. 
With all these facts, Theorem 2.3 is immediate:

Proof of Theorem 2.3 By Corollary 3.5 and Lemma 3.3, the map $f: \mathbb{R}^{n} \backslash 0 \rightarrow \mathbb{R}^{n} \backslash 0$ is bijective. Hence it is a global $C^{1}$ diffeomorphism by the usual local inverse function theorem. Furthermore, the map $f: \mathbb{R}^{n} \rightarrow \mathbb{R}^{n}$ is also bijective since $f(0)=0$, hence the global inverse $f^{-1}: \mathbb{R}^{n} \rightarrow \mathbb{R}^{n}$ exists and is continuous by Lemma 3.4. Since $f: \mathbb{R}^{n} \rightarrow \mathbb{R}^{n}$ is also continuous, it is a homeomorphism.

Acknowledgments The authors would like to thank Professor Adi Adimurthi for valuable remarks on the first version of our manuscript, leading to its considerable improvement.

Open Access This article is distributed under the terms of the Creative Commons Attribution License which permits any use, distribution, and reproduction in any medium, provided the original author(s) and the source are credited.

\section{References}

1. Asada, K., Fujiwara, D.: On some oscillatory integral transformations in $L^{2}\left(R^{n}\right)$. Jpn. J. Math. 4(2), 299-361 (1978)

2. Gordon, W.B.: On the diffeomorphisms of Euclidean space. Am. Math. Mon. 79, 755-759 (1972)

3. Hadamard, J.: Sur les correspondances ponctuelles. Paris: Oeuvres, Editions du Centre Nationale de la Researche Scientifique, pp. 383-384 (1998)

4. Hadamard, J.: Sur les transformations planes. C. R. Math. Acad. Sci. Paris 142, 74 (1906)

5. Hadamard, J.: Sur les transformations ponctuelles. Bull. Soc. Math. Fr. 34, 71-84 (1906)

6. Nijenhuis, A., Richardson Jr, R.W.: A theorem on maps with non-negative Jacobians. Mich. Math. J. 9, 173-176 (1962)

7. Ruzhansky, M., Sugimoto, M.: Global $L^{2}$-boundedness theorems for a class of Fourier integral operators. Commun. Partial Differ. Equ. 31(4-6), 547-569 (2006)

8. Ruzhansky, M., Sugimoto, M.: A smoothing property of Schrödinger equations in the critical case. Math. Ann. 335(3), 645-673 (2006) 